\title{
Love Dynamical Models with Delay
}

\author{
Kaori Saito1, Shiho Takagi2, Yoshihiro Hamaya ${ }^{3}$ \\ ${ }^{1}$ Department of Industrial Information, Iwate Prefectural University Miyako College, Miyako, Japan \\ ${ }^{2}$ Global Education Development Center, Okayama University of Science 1-1 Ridai-Cho, Okayama, Japan \\ ${ }^{3}$ Department of Information Science, Okayama University of Science 1-1 Ridai-Cho, Okayama, Japan \\ Email: saito_k@iwate-pu.ac.jp, takagi@ped.ous.ac.jp, hamaya@mis.ous.ac.jp
}

How to cite this paper: Saito, K., Takagi, S. and Hamaya, Y. (2020) Love Dynamical Models with Delay. Advances in Pure Mathematics, 10, 297-311. https://doi.org/10.4236/apm.2020.105017

Received: March 27, 2020

Accepted: May 11, 2020

Published: May 14, 2020

Copyright (C) 2020 by author(s) and Scientific Research Publishing Inc. This work is licensed under the Creative Commons Attribution International License (CC BY 4.0).

http://creativecommons.org/licenses/by/4.0/ (c) (i)

\begin{abstract}
A sufficient condition for the asymptotic stability of the equilibrium point of a system, which appears as a model for couple of the love affair with time delay, is obtained by applying the technique of linearized method and Hopfbifurcation.
\end{abstract}

\section{Keywords}

Love Affairs, Asymptotic Stability, Linearized Method, Hop-Bifurcation, Delay Dynamical Model

\section{Introduction}

In a pioneering paper [1] and a famous book [2], Strogatz considered a simple pedagogical model describing a love affair. He treated harmonic oscillation phenomena using a topic that is already on the minds of many college students, which is the time evolution of a love affair between a couple. Later, Sprott [3] proposed more realistic nonlinear triangle models for love dynamics (cf. [4] [5]). Moreover, Rinaldi who is an authority in this area, has studied several types of models describing love affairs and published many papers (cf. [6] [7] [8] [9] [10]). They treated the technique of standard linearized method. On the other hand, we study the effect of time delay on the nonlinear dynamical model describing a love affair with feedback between two individuals.

In this paper, we consider the following delay differential equation with feedback of

$$
\begin{aligned}
& \frac{\mathrm{d} R(t)}{\mathrm{d} t}=-d_{R} R(t)+f\left(J\left(t-\tau_{2}\right)\right)+\gamma_{1} A_{2}, \\
& \frac{\mathrm{d} J(t)}{\mathrm{d} t}=-d_{J} J(t)+r_{J} R\left(t-\tau_{1}\right)+\gamma_{2} A_{1}, \quad t>0,
\end{aligned}
$$


where we denote measures of the love of individuals $R(t)$ and $J(t)$ for the partner by $J(t)$ and $R(t)$ at time $t$ (like a Romeo's love or hate if negative for Juliet at time $t$ and like Juliet's love for Romeo). The parameter $d_{R}$ and $d_{J}$ are the respective decay rates in the forgetting coefficient. The $r_{J}$ is the return rates for $R(t)$ and it describes the direct effect of his love on the partner $J(t)$. $A_{1}, A_{2}$ are constant coefficients reflecting the appeal of Romeo and Juliet, respectively and $\gamma_{1}$ is Romeo's reaction rate to Juliet's appeal and $\gamma_{2}$ is reaction of Juliet to Romeo's appeal. $\tau_{1} \geq 0$ and $\tau_{2} \geq 0$ are nonnegative delay terms. Since $R(t)$ and $J(t)$ are each emotions at time $t$, naturally, it later seeks for the conditions that the solution $(R(t), J(t))$ of Equation (1) exists, whenever the initial date is given and all coefficients are positive numbers. To do this, we assume the monotone bounded and continuously differentiable function $f(J)$.

Equation (1) is an extending model of the without delay differential equation

$$
\begin{gathered}
\frac{\mathrm{d} R(t)}{\mathrm{d} t}=-d_{R} R(t)+r_{R} J(t)+\gamma_{1} A_{2}, \\
\frac{\mathrm{d} J(t)}{\mathrm{d} t}=-d_{J} J(t)+r_{J} R(t)+\gamma_{2} A_{1},
\end{gathered}
$$

which has been proposed by Rinaldi [7] and Rinaldi et al. [11] as a model for the linear system of love dynamics, where $r_{R}$ describes the direct effect to her love on the partner $R(t)$. Next, we introduce another differential model of love with delay

$$
\begin{aligned}
& \frac{\mathrm{d} R(t)}{\mathrm{d} t}=-d_{R} R(t)+H_{1}(J(t-\tau))+\gamma_{1} A_{2}, \\
& \frac{\mathrm{d} J(t)}{\mathrm{d} t}=-d_{J} J(t)+H_{2}(R(t-\tau))+\gamma_{2} A_{1},
\end{aligned}
$$

proposed by Liao and Ran [12] and Son and Park [13], where $H_{i}(x),(i=1,2)$ are the little bit strong restricted functions with same delay $\tau$. To consider more reality love regime than ordinary differential system (2), they investigate that the stable equilibrium point is destabilized for a delay larger than a threshold value and then bifurcates to a limit cycle via a Hopf bifurcation when Romeo is secure and Juliet is non-secure.

We investigate the first problem how is the condition of the asymptotically stable of the equilibrium point of Equation (1). Moreover, we have second one what is the oscillatory criteria of Equation (1) and, we should consider a simple example for our Equation (1).

Our first goal is to give the asymptotic stability of equilibrium points of 2-dimensional dynamics of Romeo and Juliet in the multiple equilibrium case, using linearized method. The second case we take up concerns the romantic real style of Romeo and Juliet with 2-difference time delays, using a technique of Hopf bifurcation. Moreover, we consider the oscillation criteria of (1) without constant terms and simple examples of the linearized equation of (1).

We can show that the existence of solution $(R(t), J(t))$ is guaranteed for Equation (1) whenever the initial conditions are bounded continuous functions; 


$$
\begin{aligned}
& R(s)=\phi_{1}(s) \text { for }-\tau_{1} \leq s \leq 0, \\
& \text { and } \\
& J(s)=\phi_{2}(s) \text { for }-\tau_{2} \leq s \leq 0,
\end{aligned}
$$

where $\phi_{i}(s) \in C\left(\left[-\tau_{i}, 0\right], R\right)$ (in short, $C$ ) and $\phi_{i}(0) \geq 0$ for $i=1,2$. Here, Banach space $C(I, R)$ is the set of all continuous functions mapping $I$ into $R$ with supremum norm defined by $|\cdot|_{C}$ (in short, $\left.|\cdot|\right)$, where $|\phi|=\sup _{s \in[-r, 0]}|\phi(s)|, \phi \in C$.

\section{Stability Criteria of Equilibrium Points}

In this section we study the stability of equilibrium points of Equation (1). We have the equilibrium point $E^{*}=E^{*}\left(R^{*}, J^{*}\right)$ of Equation (1), where

$$
\begin{aligned}
& R^{*}=\frac{d_{J} J^{*}-\gamma_{2} A_{1}}{r_{J}} \text { and } \\
& f\left(J^{*}\right)=d_{R} R^{*}-\gamma_{1} A_{2}=\frac{d_{R} d_{J} J^{*}-d_{R} \gamma_{2} A_{1}-r_{J} \gamma_{1} A_{2}}{r_{J}} .
\end{aligned}
$$

We investigate the stability of the equilibrium point $E^{*}=E^{*}\left(R^{*}, J^{*}\right)$ by linearization. Let

$$
\begin{aligned}
& R(t)=R^{*}+x(t), \\
& J(t)=J^{*}+y(t),
\end{aligned}
$$

where $x(t)$ and $y(t)$ are small perturbations. Then, the linearized form of the Equation (1) about the equilibrium point $E^{*}$ is writing $x(t), y(t)$ for $\dot{x}(t)$ and $\dot{y}(t)$.

$$
\begin{aligned}
& \dot{x}(t)=-d_{R} x(t)+f^{\prime}\left(J^{*}\right) y\left(t-\tau_{2}\right), \\
& \dot{y}(t)=-d_{J} y(t)+r_{J} x\left(t-\tau_{1}\right) .
\end{aligned}
$$

Remark 1. We consider $f(J)$ are particular forms by taken as two cases: for some odd integer $l \geq 1$,

$$
\left(\mathrm{H}_{1}\right) \quad f(J)=\frac{r_{R} J^{l}}{K+J^{l}} \text { for } J \geq 0
$$

and

$$
\left(\mathrm{H}_{2}\right) \quad f(J)=r_{R} \tanh \left(\frac{J}{J_{0}}\right),
$$

where $K>0$ is a real number and $J_{0}$ is the concentration parameters related to the switching of the love individual by a Juliet's love function $J(t)$. For $l=1$, the function $f$ in $\left(\mathrm{H}_{1}\right)$ is considered by [6] and the function $f$ of $\left(\mathrm{H}_{2}\right)$ is treated in [14]. It seems that $\left(\mathrm{H}_{2}\right)$ is a more adjust condition than $\left(\mathrm{H}_{1}\right)$ as situation of love affairs. So, in this paper, we mainly employ the condition $\left(\mathrm{H}_{2}\right)$.

In the case where $\left(\mathrm{H}_{1}\right)$ and $\left(\mathrm{H}_{2}\right)$, respectively, $J^{*}$ is given by the solution of the equation

$$
\left(J^{*}\right)^{l+1}-\frac{r_{R} r_{J}+A}{d_{R} d_{J}}\left(J^{*}\right)^{l}+K J^{*}-\frac{K A}{d_{R} d_{J}}=0
$$


and

$$
\tanh \left(\frac{J^{*}}{J_{0}}\right)-\frac{d_{R} d_{J}}{r_{R} r_{J}} J^{*}+\frac{A}{r_{R} r_{J}}=0,
$$

where $A=d_{R} \gamma_{2} A_{1}+r_{J} \gamma_{1} A_{2}$. In Equation (5), the case where each assumption $\left(\mathrm{H}_{1}\right)$ and $\left(\mathrm{H}_{2}\right)$, we have

$$
f^{\prime}\left(J^{*}\right)=\frac{K l r_{R}\left(J^{*}\right)^{l-1}}{\left(K+\left(J^{*}\right)^{l}\right)^{2}},\left(\text { by } \mathrm{H}_{1}\right)
$$

and

$$
f^{\prime}\left(J^{*}\right)=\frac{r_{R} / J_{0}}{\cos ^{2} h\left(J^{*} / J_{0}\right)},\left(\text { by } \mathrm{H}_{2}\right),
$$

respectively.

We can show the next theorem by using Routh-Hurwitz theorem (cf. [2] [11] and [15]) for the second-order differential equation.

The stability results in this article are the following.

Theorem 1 (without delay case). (cf. [11]). Suppose that

$$
d_{R}+d_{J}>0 \text { and } d_{R} d_{J}>r_{J} f^{\prime}\left(J^{*}\right) \text {. }
$$

Then, the equilibrium point $E^{*}$ of Equation (1) with $\tau_{1}=\tau_{2}=0$ is asymptotically stable.

Theorem 2 (with delay case). The necessary and sufficient condition for the asymptotic stability of the equilibrium point $E^{*}$ of Equation (1) with all delay $\tau>0$ is the condition (6).

Proof. To prove this theorem, we apply the approach of (Theorem 3.7.3 in [16]). When delays $\tau_{1}, \tau_{2} \neq 0$, the characteristic equation associated with (5) can be written as

$$
D(\lambda, \tau)=\lambda^{2}+a \lambda+b+c \mathrm{e}^{-\tau \lambda}=0
$$

where

$$
\begin{aligned}
& a=d_{R}+d_{J}, \\
& b=d_{R} d_{J} \text { and } \\
& c=-r_{J} f^{\prime}\left(J^{*}\right),
\end{aligned}
$$

and $\tau=\tau_{1}+\tau_{2}$. It is easy to verify the necessity of the condition (6). For instance, if (6) does not hold then the trivial solution of (5) is not asymptotically stable for $\tau=0$, from the proof of Theorem 1. If a real number $z$ and a $\tau \geq 0$ exist such that $D(i z, \tau)=0$ then for such $\tau$, the characteristic Equation (7) has a pair of pure imaginary roots and hence the trivial solution of (5) is not asymptotically stable.

Setting $\lambda=\mu+i v$ in (7) and separating the real and imaginary parts, we get a system of transcendental equations:

$$
\mu^{2}-v^{2}+a \mu+b+c \mathrm{e}^{-\mu \tau} \cos v \tau=0,
$$




$$
2 \mu \nu+a v-c \mathrm{e}^{-\mu \tau} \sin v \tau=0 .
$$

Here, in formula (9), the variables of the trigonometric functions are covered with (7) and (8). One can write (7) in the form

$$
\lambda^{2}+a \lambda+w=0,
$$

where $w=b+c \mathrm{e}^{-\lambda \tau}$. For any real $z$, and $\tau \geq 0$, we have

$$
D(i z, \tau)=-z^{2}+a i z+b+c \mathrm{e}^{-i z \tau} .
$$

Then, for $z=0, D(i z, \tau)=b+c \neq 0$ by (6) and (8). For $z \neq 0$, let us suppose $\tau$ varies on the interval $\left[0, \frac{2 \pi}{|z|}\right]$ implying that $|z \tau|$ will vary in $[0,2 \pi]$. This means that $\mathrm{e}^{\mathrm{iz \tau}}$ will vary over unit circle. Thus we can let for $z \neq 0, \quad z \tau$ to be another independent variable $\sigma$ (where $\sigma=-z \tau$ ). We can write

$$
H(z, \sigma)=G(z, \sigma)+i K(z, \sigma)=\left(-z^{2}+b+c \cos \sigma\right)+i(a z+c \sin \sigma) .
$$

Thus,

$$
\begin{gathered}
G(z, \sigma)=-z^{2}+b+c \cos \sigma=0, \\
K(z, \sigma)=a z+c \sin \sigma=0 .
\end{gathered}
$$

Here, eliminating $\sigma$ from (11) and (12), we get

$$
U(z)=z^{4}+\left(a^{2}-2 b\right) z^{2}+\left(b^{2}-c^{2}\right)=0 .
$$

A necessary and sufficient condition for $U(z)=0$ not to have non-zero real root is $b^{2}-c^{2} \geq 0$, that is, $b \geq c$ from (6). If $U(z)=0$ has non-zero real root, then $b^{2}-c^{2}<0$. From (11) and (12), we have

$$
\tan \sigma=\frac{-a z}{z^{2}-b} .
$$

Then, we obtain the real values of $\sigma$ which satisfy (11) and (12). Thus, a set of necessary and sufficient condition for the asymptotic stability of the interior equilibrium is $c<b$. This completes the proof of Theorem 2 .

Remark 2. The above Theorem 1 and 2 hold for the both functions $\left(\mathrm{H}_{1}\right)$ and $\left(\mathrm{H}_{2}\right)$. This talk is motivated by Das et al. [17] [18] and Hamaya et al. [19], that is "Study the stability and the existence of almost periodic solutions of the Equation (5)", and we also regard Theorem 1, 2 and next Theorem 3, 4 as a partial answer in the affirmative for their research.

For the more complicated equation of (3), [4] [10] [11] and [20] have shown the asymptotic stability of the equilibrium point $E^{*}$ under the more complicated conditions using a bifurcation technique and others.

\section{Estimation for the Length of Delay to Preserve Stability and Bifurcation Results}

In this section, we suppose that in the absence of delay $E^{*}\left(R^{*}, J^{*}\right)$ is asymptotically stable. This is guaranteed if (6) holds. By continuity of solutions and for sufficiently small $\tau=\tau_{1}+\tau_{2}>0$, all eigenvalues of (7) have negative real parts 
provided that no eigenvalue bifurcates from $+\infty$, which could happen since this is a retarded delay system. It is then possible to use a criterion of Nyquist which we describe below to estimate the range of $\tau$ for which $E^{*}$ remains asymptotically stable. Here we follow the approach by [16] [17] [18] for such estimation of $\tau$. We consider the system (5) and the space of real valued continuous functions defined on $C[-\tau, \infty)$ satisfying the initial conditions (4).

Theorem 3. If

$$
d_{R}+d_{J}>d_{R} d_{J}-r_{J} f^{\prime}\left(J^{*}\right) \geq 0,
$$

then there exists a $\tau_{+}$given by

$$
\tau_{+}=\frac{-c+\sqrt{c^{2}+2 c v_{+}^{2}(a-b-c)}}{c v_{+}^{2}}, \text { where } a-b-c>0,
$$

such that for all $\tau<\tau_{+}$, the equilibrium point $E^{*}$ of (5) is asymptotically stable.

Proof. Let $\bar{x}(W), \bar{y}(W)$ be the Laplace transform of $x(t)$ and $y(t)$, respectively. Taking the Laplace transform of (5), we have

$$
\begin{aligned}
& (W-\alpha) \bar{x}(W)=\beta \mathrm{e}^{-W \tau_{2}} \bar{y}(W)+\beta \mathrm{e}^{-W \tau_{2}} K_{1}(W)+x(0), \\
& (W-\delta) \bar{y}(W)=\gamma \mathrm{e}^{-W \tau_{1}} \bar{x}(W)+\gamma \mathrm{e}^{-W \tau_{1}} K_{2}(W)+y(0),
\end{aligned}
$$

where

$$
K_{1}(W)=\int_{-\tau_{2}}^{0} \mathrm{e}^{-W t} y(t) \mathrm{d} t, \quad K_{2}(W)=\int_{-\tau_{1}}^{0} \mathrm{e}^{-W t} x(t) \mathrm{d} t,
$$

and

$$
\begin{aligned}
& \alpha=-d_{R}, \quad \delta=-d_{J}, \\
& \beta=f^{\prime}\left(J^{*}\right), \quad \gamma=r_{J} .
\end{aligned}
$$

Rearranging, we have

$$
\begin{aligned}
& {\left[W^{2}-W(\alpha+\delta)+\alpha \delta-\beta \gamma \mathrm{e}^{-W\left(\tau_{1}+\tau_{2}\right)}\right] \bar{x}(W)} \\
& =\beta \mathrm{e}^{-W \tau_{2}} y(0)+\beta \gamma \mathrm{e}^{-W\left(\tau_{1}+\tau_{2}\right)} K_{2}(W)+(W-\delta) x(0)+(W-\delta) \beta \mathrm{e}^{-W \tau_{2}} K_{1}(W) .
\end{aligned}
$$

The inverse Laplace transform of $\bar{x}(W)$ will have terms which exponentially increase with time, if $\bar{x}(W)$ has poles with positive real parts. For $E^{*}$ to be locally asymptotically stable, it is necessary and sufficient that all poles of $\bar{x}(W)$ have negative real parts. We shall employ the Nyquist criterion which states that if $W$ is arc length of a curve encircling the right half plane, the curve $\bar{x}(W)$ will encircle the origin a number of times equal to the difference between the number of poles and the number of zeros of $\bar{x}(W)$ in the right half plane. We see that the conditions for the local asymptotically stability of $E^{*}$ is given by

$$
\begin{aligned}
& \operatorname{Im}\left\{S\left(i v_{0}\right)\right\}>0, \\
& \operatorname{Re}\left\{S\left(i v_{0}\right)\right\}=0,
\end{aligned}
$$

where $S(W)=W^{2}+a W+b+c \mathrm{e}^{-\lambda \tau}$ and $v_{0}$ is the smallest positive root of the Equation (14), where $a, b, c$ are numbers in (7). 
In our case, these conditions become

$$
\begin{gathered}
a v_{0}>c \sin v_{0} \tau, \\
-v_{0}^{2}+b=-c \cos v_{0} \tau .
\end{gathered}
$$

To get our estimate on the length of delay, we recall conditions

$$
\begin{gathered}
a v>c \sin v \tau, \\
-v^{2}+b=-c \cos v \tau,
\end{gathered}
$$

and $E^{*}$ is stable if the inequality (15) holds at $v=v_{0}$, when $v_{0}$ is the first positive root of Equation (16). Our technique will be to find an upper bound $v_{+}$ on $v_{0}$ independent of $\tau$ and then to estimate $\tau$ so that (15) holds for all values of $v, 0 \leq v \leq v_{+}$, hence in particular at $v=v_{0}$.

The unique positive solution of $v^{2}-b-c=0$, denoted by $v_{+}$is always greater than or equal to $v_{0}$. Then, we have

$$
v_{+}=\sqrt{b+c} \text {. }
$$

From (15),

$$
0<a-\frac{c}{v} \sin v \tau .
$$

At $\tau_{1}=0$ and $\tau_{2}=0, a>0$ and $\tau_{i}=0(i=1,2)$ in Equation (16) gives

$$
v^{2}=b+c<a
$$

Hence, (17) is valid when $\tau=0$, so by continuity, it continues to hold for small enough $\tau>0$ at $v=v_{0}$. Now, by substituting $v^{2}$ from (16) into (17), we get

$$
v^{2}=b+c \cos v \tau<a-\frac{c}{v} \sin v \tau \text {. }
$$

Thus,

$$
c(\cos v \tau-1)+\frac{c}{v} \sin v \tau<a-b-c .
$$

Let us define $\phi(\tau, v)=c(\cos v \tau-1)+\frac{c}{v} \sin v \tau$ and we set $\eta=a-(b+c)$.

Using the estimates $\sin v \tau \leq v \tau$ and $1-\cos v \tau \leq \frac{1}{2} \nu^{2} \tau^{2}$, we obtain

$$
\phi(\tau, v) \leq \psi(\tau, v)=\frac{1}{2} c v^{2} \tau^{2}+c \tau \leq \psi\left(\tau, v_{+}\right) .
$$

Now, if $\psi\left(\tau, v_{+}\right)<\eta$, then $\phi\left(\tau, v_{0}\right)<\eta$. Let $\tau_{+}$denote the unique positive root of $\psi\left(\tau, v_{+}\right)=\eta$. Then, we have

$$
\frac{1}{2} c v_{+}^{2} \tau^{2}+c \tau-(a-b-c)=0 .
$$

Thus,

$$
\tau_{+}=\frac{-c+\sqrt{c^{2}+2 c v_{+}^{2}(a-b-c)}}{c v_{+}^{2}},
$$

where $a-b-c>0$. 
Then, for $\tau<\tau_{+}$, the Nyquist criteria holds and $\tau_{+}$is the estimate for the length of the delay $\tau$ for which stability is preserved. Thus, the proof of this theorem completes.

Theorem 4. If we set $P_{2}=\left(d_{R} d_{J}\right)^{2}-\left[r_{J} f^{\prime}\left(J^{*}\right)\right]^{2}<0$ and if $E^{*}$ is unstable for $\tau=0$, then it remains unstable for $\tau>0$. Moreover, if $P_{2}<0$ and if $E^{*}$ is asymptotically stable for $\tau=0$, then it is impossible that it remains stable for all $\tau>0$.

Hence, there exists a $\hat{\tau}>0$ such that, for $\tau<\hat{\tau}$, the equilibrium point $E^{*}$ is asymptotically stable and for $\tau>\hat{\tau}$, the equilibrium point $E^{*}$ is unstable and moreover, as $\tau$ increases through $\hat{\tau}, E^{*}$ bifurcates into small amplitude periodic solutions of Hopf type [4] [13]. The existence of unique $\hat{\tau}$ is given by

$$
\hat{\tau}=\frac{1}{\hat{v}} \tan ^{-1}\left(\frac{a \hat{v}}{\hat{v}^{2}-b}\right)+\frac{n \pi}{\hat{v}}, \quad n=0,1,2, \cdots .
$$

Our required $\hat{\tau}$ is given by $n=0$ in (18) and hence the Hopf-bifurcation criteria are satisfied.

Proof. Let us consider $\lambda$ and hence $\mu$ and $v$ as a function of $\tau$. We are interested in the change of stability of equilibrium point $E^{*}\left(R^{*}, J^{*}\right)$ which occurs at the values of $\tau$ for which $\mu=0$ and $v \neq 0$, that is $b+c \neq 0$ by (9). Let $\hat{\tau}$ be such that $\mu(\hat{\tau})=0$ and $v(\hat{\tau})=\hat{v} \neq 0$. Then (9) and (10) become

$$
\begin{gathered}
-\hat{v}^{2}+b+c \cos \hat{\tau} \hat{v}=0, \\
a \hat{v}-c \sin \hat{\tau} \hat{v}=0 .
\end{gathered}
$$

From the above equations, we get

$$
\hat{v}^{4}+\left(a^{2}-2 b\right) \hat{v}^{2}+\left(b^{2}-c^{2}\right)=0 .
$$

To analyze the change in the behavior of the stability of $E^{*}$ with respect to $\tau$, we examine the sign of $\mathrm{d} \mu / \mathrm{d} \tau$ as $\mu$ crosses zero, that is we analyze the sign of $\mathrm{d} \mu(\hat{\tau}) / \mathrm{d} \tau$ where $\mu(\hat{\tau})=0$. If this derivative is positive (negative) then clearly a stabilization (destabilization) can not take place at that value of $\tau$. We differentiate Equation (9) and Equation (10) with respect to $\tau$. Then setting $\tau=\hat{\tau}, \mu=0$ and $v=\hat{v}$, we get

$$
\xi \frac{\mathrm{d} \mu(\hat{\tau})}{\mathrm{d} \tau}+\eta \frac{\mathrm{d} v(\hat{\tau})}{\mathrm{d} \tau}=\alpha, \quad-\eta \frac{\mathrm{d} \mu(\hat{\tau})}{\mathrm{d} \tau}+\xi \frac{\mathrm{d} v(\hat{\tau})}{\mathrm{d} \tau}=\beta
$$

where

$$
\begin{gathered}
\xi=a-c \hat{\tau} \cos \hat{\tau} \hat{v}, \\
\eta=-2 \hat{v}-c \hat{\tau} \sin \hat{\tau} \hat{v}, \\
\alpha=c \hat{v} \sin \hat{\tau} \hat{v} \quad \text { and } \quad \beta=c \hat{v} \cos \hat{\tau} \hat{v} .
\end{gathered}
$$

We have

$$
\left(\xi^{2}+\eta^{2}\right) \frac{\mathrm{d} \mu(\hat{\tau})}{\mathrm{d} \tau}=\xi \alpha-\eta \beta
$$

Thus, 


$$
\frac{\mathrm{d} \mu(\hat{\tau})}{\mathrm{d} \tau}=\frac{\xi \alpha-\eta \beta}{\xi^{2}+\eta^{2}}
$$

$\frac{\mathrm{d} \mu(\hat{\tau})}{\mathrm{d} \tau}$ has the same sign as $\xi \alpha-\eta \beta$. Now,

$$
\begin{aligned}
\xi \alpha-\eta \beta & =(a-c \hat{\tau} \cos \hat{\tau} \hat{v}) c \hat{v} \sin \hat{\tau} \hat{v}-(-2 \hat{v}-c \hat{\tau} \sin \hat{\tau} \hat{v}) c \hat{v} \cos \hat{\tau} \hat{v} \\
& =a c \hat{v} \sin \hat{\tau} \hat{v}+2 c \hat{v}^{2} \cos \hat{\tau} \hat{v} .
\end{aligned}
$$

Substituting the values of $\sin \hat{\tau} \hat{v}$ and $\cos \hat{\tau} \hat{v}$ from (19) and (20), we get

$$
\xi \alpha-\eta \beta=\hat{v}^{2}\left[2 \hat{v}^{2}+\left(a^{2}-2 b\right)\right] .
$$

Let

$$
\phi(z)=z^{2}+P_{1} z+P_{2}
$$

where

$$
\begin{gathered}
P_{1}=a^{2}-2 b=d_{R}^{2}+d_{J}^{2}, \\
P_{2}=b^{2}-c^{2}=\left(d_{R} d_{J}\right)^{2}-\left[r_{J}\left\{f^{\prime}\left(J^{*}\right)\right\}\right]^{2} .
\end{gathered}
$$

Now, $\phi(z)$ is the solution of (21) with $\hat{v}^{2}=z$. Then, we have

$$
\frac{\mathrm{d} \phi\left(\hat{v}^{2}\right)}{\mathrm{d} z}=2 \hat{v}^{2}+P_{1}=2 \hat{v}^{2}+\left(a^{2}-2 b\right)=\frac{\xi \alpha-\eta \beta}{\hat{v}^{2}} .
$$

Then,

$$
\frac{\mathrm{d} \phi\left(\hat{v}^{2}\right)}{\mathrm{d} z}=\frac{\xi^{2}+\eta^{2}}{\hat{v}^{2}} \cdot \frac{\mathrm{d} \mu(\hat{\tau})}{\mathrm{d} \tau}
$$

Thus, we have

$$
\frac{\mathrm{d} \mu(\hat{\tau})}{\mathrm{d} \tau}=\frac{\hat{v}^{2}}{\xi^{2}+\eta^{2}} \cdot \frac{\mathrm{d} \phi\left(\hat{v}^{2}\right)}{\mathrm{d} z} .
$$

Therefore, the criteria for preservation of instability (stability) of $E^{*}\left(R^{*}, J^{*}\right)$ has the following cases;

1) If the polynomial $\phi(z)$ has no positive root (being contradictory to the existence of $\hat{v}>0$ be real) there can be no change of stability.

2) If $\phi(z)$ is increasing (decreasing) at all of its positive roots, instability (stability) is preserved.

Now, in this case,

$\left(C_{1}\right)$ If $P_{2}<0, \phi(z)$ has a unique positive real root, then it must increase at that point. Because, $\phi(z)$ is a cubic in $z, \lim _{z \rightarrow \infty} \phi(z)=\infty$.

$\left(C_{2}\right)$ If $P_{2}>0$, then $\left(C_{1}\right)$ is satisfied, that is there can be no change of stability. From (19) and (20), (18) is satisfied. This completes the proof of Theorem 4.

\section{Oscillatory Criteria}

We study the oscillatory behavior of the linearized system (1) involving two distinct delays which are different. But, so far as the author's knowledge goes, there are very few studies on the analysis of oscillation of model with unequal delays. 
To make the study mathematically tractable, all the delays are assumed to be equal and equal to the $1 / 2$ of the sum of all the delays. From physiological date, it's not psychology, today delay is nearly 28 - 30 hours in [18], from the numerical simulation of the linearized system, it is seen that the pulsated or oscillatory behavior is present, if the individual unequal delay exceed from 5 hours to two days. For simplicity, without much loss of generality, we assume that $\tau_{1}=\tau_{2}=\tau$, where $\tau_{i}>5(i=1,2)$. Then the system (5) can be written as

$$
\begin{aligned}
& \dot{x}(t)=-d_{R} x(t)+f^{\prime}\left(J^{*}\right) y(t-\tau), \\
& \dot{y}(t)=-d_{J} y(t)+r_{J} x(t-\tau),
\end{aligned}
$$

where $d_{R}>0, d_{J}>0, \tau>0, f^{\prime}\left(J^{*}\right)>0$ and $r_{J}>0$. We will find a set of sufficient conditions for all bounded solutions of the linearized system (22) to be oscillatory when the system has equal multi delays (cf. [16]). Here we adopt the following definition.

Definition 1. A nontrivial vector $u=\{x(t), y(t)\}^{\mathrm{T}}$ defined on $[0, \alpha)$, some $\alpha>0$, is said to be oscillatory, if and only if at least one component of $x(t)$ has arbitrary large zeros on $[0, \alpha)$.

Let us define

$$
\begin{gathered}
\max \left\{f^{\prime}\left(J^{*}\right), r_{J}, 1\right\}=\gamma>0, \text { and } \\
\max \left\{-d_{R},-d_{J}\right\}=-\tilde{d}<0 .
\end{gathered}
$$

Theorem 5. We assume the following conditions:

$$
\text { (i) } \gamma>\tilde{d} \text {, and (ii) } \mathrm{e}^{\tau \tilde{d}}<\gamma \tau \mathrm{e} \text {. }
$$

Then all the bounded solutions of (22) corresponding to continuous initial conditions on $[-\tau, 0]$ are oscillatory on $[0, \infty)$.

Proof. Suppose that there exists a solution $u=\{x(t), y(t)\}^{\mathrm{T}}$ of (22), which is bounded and non oscillatory on $[0, \infty)$.

Then, it follows that there exists a $t^{*}>0$ such that no component of $x(t)$ has a zero for $t>t^{*}+\tau$, and as a consequence, we have

$$
\begin{gathered}
|\dot{x}(t)| \geq-d_{R}|x(t)|+f^{\prime}\left(J^{*}\right)|y(t-\tau)|, \\
|\dot{y}(t)| \geq-d_{J}|y(t)|+r_{J}|x(t-\tau)|, \text { for } t>t^{*}+2 \tau .
\end{gathered}
$$

Let $u(t)=|x(t)|+|y(t)|>0$, for $t \geq t^{*}+\tau$. Thus, we get $\dot{u}(t) \geq-\tilde{d} u(t)+\gamma u(t-\tau)$ for $t \geq t^{*}+2 \tau$. Now, we consider the scalar delay differential equation

$$
\dot{v}(t)=-\tilde{d} v(t)+\gamma v(t-\tau) \text {, for } t \geq t^{*}+2 \tau \text {, with } v(s)=u(s), s \in\left[t^{*}, t^{*}+\tau\right] .
$$

Using the comparison theorem in [16], we have

$$
u(t) \leq v(t), \text { for } t \geq t^{*}+2 \tau .
$$

We now claim that all bounded solutions of (24) are oscillatory on $\left[t^{*}+2 \tau, \infty\right)$. Suppose that this is not the case, then the characteristic equation associated with (24) is given by 


$$
\lambda=-\tilde{d}+\gamma \mathrm{e}^{-\lambda \tau},
$$

has a non positive root, we say, $\lambda^{*}<0$ and it follows from (i) of (23) that $\lambda^{*} \neq 0$, then $\lambda^{*}<0$, and hence, we have

$$
\left|\lambda^{*}\right| \geq-\tilde{d}+\gamma \mathrm{e}^{\left|\lambda^{*}\right| \tau}
$$

Then, $\left|\lambda^{*}\right|+\tilde{d} \geq \gamma \mathrm{e}^{\left|\lambda^{*}\right|_{\tau}^{\tau}}$, and by the expansion into series of $\mathrm{e}^{X}$ for some $X \in \mathbf{R}$, it is clear that $\mathrm{e}^{\left(\left|\lambda^{*}\right|+\tilde{d}\right) \tau-1} \geq \tau\left(\left|\lambda^{*}\right|+\tilde{d}\right)$. Thus, we get

$$
1 \geq \frac{\gamma e^{\left|\lambda^{*}\right| \tau}}{\left|\lambda^{*}\right|+\tilde{d}} \geq \gamma \tau \mathrm{e}^{1-\tilde{d} \tau} .
$$

The local inequality contradicts (ii) of (23), and hence, our claim regarding the oscillatory nature of $v$ on $[0, \infty)$ is valid. Since $v$ has arbitrarily large zeros by (25), which means that $u(t)=|x(t)|+|y(t)|$ is oscillatory implying that $\boldsymbol{x}(t)$ is oscillatory, but this is absurd. Since $\boldsymbol{x}(t)$ is taken to be non oscillatory vector. So, there cannot exist a bounded non oscillatory solution of (22) when the conditions (i) and (ii) of (23) hold, and therefore, the proof is complete.

\section{Examples}

We consider concrete examples of the following linearized equation of Equation (1)

$$
\begin{aligned}
& \dot{x}(t)=-a x(t)+b y\left(t-\mu_{2}\right), \\
& \dot{y}(t)=c x\left(t-\mu_{1}\right)-d y(t), \quad t>0,
\end{aligned}
$$

where, in Equation (5), $d_{R}=a, d_{J}=d, \tau_{1}=\mu_{1}, \tau_{2}=\mu_{2}, r_{J}=c$ and $f^{\prime}\left(J^{*}\right)=b$, and moreover, all parameters can be set $0<a, b, c, d<1$ by our assumptions for (5) and especially (22).

i) For simplicity, we set $a=1 / 2, b=1 / 3, c=1 / 3, d=1 / 2$ and $\mu_{1}=\mu_{2}=2$. Then, $d_{R}=a(=1 / 2)>r_{J}=c(=1 / 3)>0$, and $d_{J}=d(=1 / 2)>f^{\prime}\left(J^{*}\right)=b(=1 / 3)>0$. Thus, it clear satisfies assumption (6) and $\left(\mathrm{H}_{2}\right)$. Moreover, we have

$$
f(J)=(3 / 4) \tanh (J) \text { and } E^{*}=\left(R^{*}, J^{*}\right) \approx(1.2,1.0),
$$

where

$$
f^{\prime}\left(J^{*}\right)=\frac{3 / 4}{\left\{\cosh \left(J^{*}\right)\right\}^{2}}
$$

for $J_{0} \equiv 1$ in $\mathrm{H}_{2}$. The initial functions are defined by

$$
\begin{gathered}
R(\theta)=\phi_{1}(\theta) \equiv 1>0, \text { and } \\
J(\theta)=\phi_{2}(\theta) \equiv 1>0,
\end{gathered}
$$

belong to the $\phi_{i}(\theta) \in C[-2,0]$ for $i=1,2$.

From our Theorem 2, we can show that for time delay $\mu_{i}=2>0$, the zero 
solution $E_{0}$ of Equation (5) is asymptotically stable, i.e. the equilibrium pint $E^{*}$ of Equation (1) is asymptotically stable by assumptions (6) and $\left(\mathrm{H}_{2}\right)$.

ii) We also set $a=1 / 2, b=1 / 3, c=1 / 3, d=1 / 2$ and $\mu_{1}=1, \mu_{2}=5$. Then, $d_{R}=a(=1 / 2)>r_{J}=c(=1 / 3)>0$, and $d_{J}=d(=1 / 2)>f^{\prime}\left(J^{*}\right)=b(=1 / 3)>0$. Thus, it satisfies assumption $(6)$ and $\left(\mathrm{H}_{1}\right)$. We denote the initial functions by

$$
\begin{gathered}
R(\theta)=\phi_{1}(\theta) \equiv 1>0, \text { and } \\
J(\theta)=\phi_{2}(\theta) \equiv 1>0,
\end{gathered}
$$

belong to the $\phi_{1}(\theta) \in C[-1,0]$ and $\phi_{2}(\theta) \in C[-5,0]$.

By our Theorem 2, we can show that for time delays $\mu_{1}=1>0$ and $\mu_{2}=5>0$, the zero solution $E_{0}$ of Equation (5) is asymptotically stable, i.e. the equilibrium pint $E^{*}$ of Equation (1) is asymptotically stable by assumptions (6) and $\left(\mathrm{H}_{2}\right)$.

\section{Conclusions}

We got the results of Theorem 2 - 5 that the asymptotic stability of the equilibrium point $E^{*}$ and the oscillatory condition for the delay difference Equation (1), by using the technique of linearized method, the bifurcation technique and others. Moreover, we have given the simple example for Theorem 2 that the equilibrium point $E^{*}$ of Equation (5), that is Equation (1), is the asymptotically stable by assumptions (6), $\left(\mathrm{H}_{2}\right)$ and all positive delay $\tau>0$.

Figures 1-4 of the final page denote the asymptotic stability of the zero solution of Equation (26). Here, we denote measures of the love of individuals, $x=x(t)$ and $y=y(t)$ for the partner $x(t)$ and $y(t)$ at time $t$, and moreover the vertical line is time $t$.

In the case of (i) of Examples 5, the solutions of (26) approach the equilibrium point $(x(t), y(t))=(0,0)$.

Figure 2 is the phase space of $(x, y)$-plane in Figure 1.

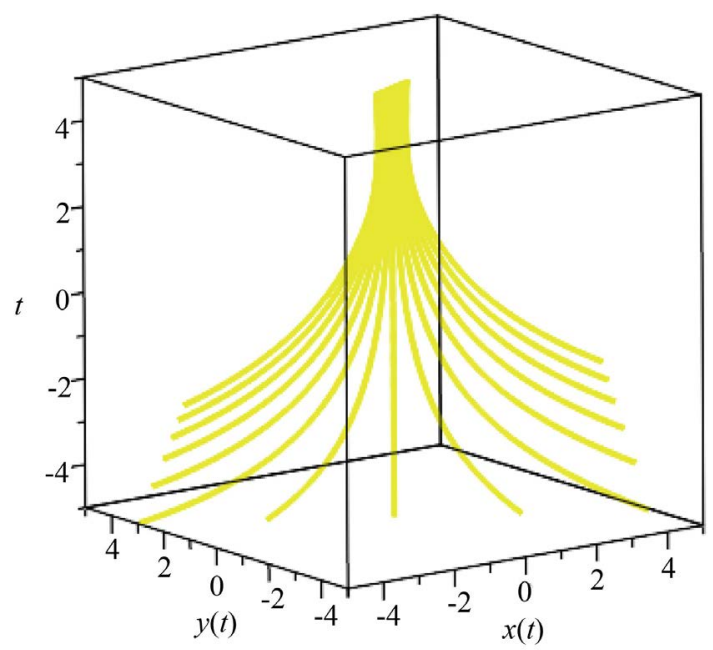

Figure 1. $(t, x(t), y(t))$. 


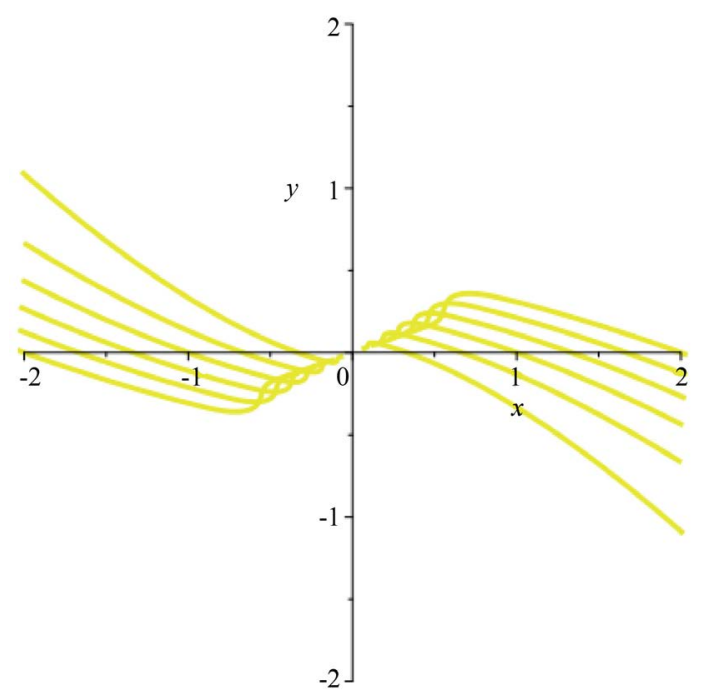

Figure 2. $(x(t), y(t))$.

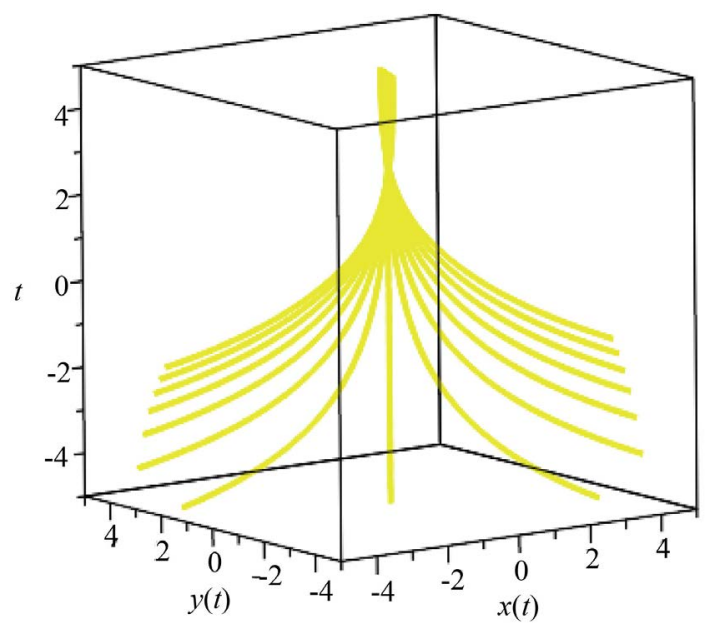

Figure 3. $(t, x(t), y(t))$.

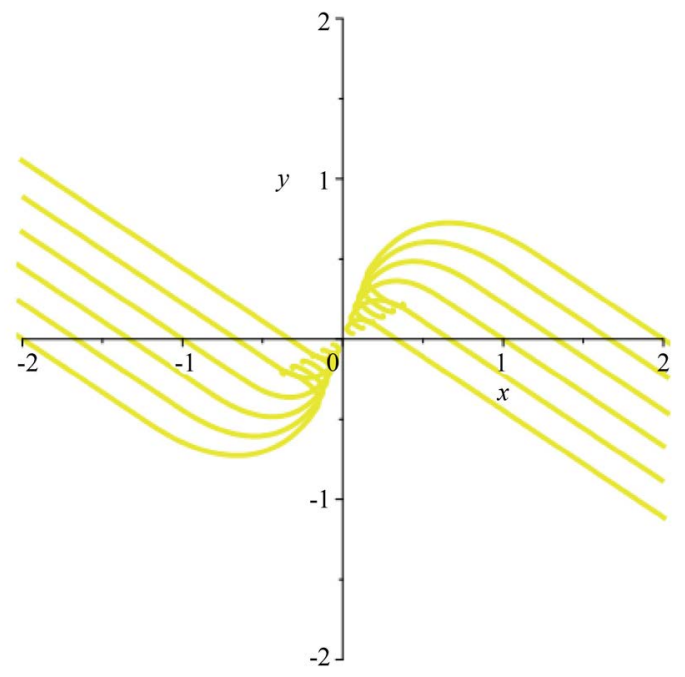

Figure 4. $(x(t), y(t))$. 
In the case of (ii) of Examples 5, the solutions of (26) approach the equilibrium point $(x(t), y(t))=(0,0)$.

Figure 4 is the phase space of $(x, y)$-plane in Figure 3.

\section{Conflicts of Interest}

The authors declare no conflicts of interest regarding the publication of this paper.

\section{References}

[1] Strogatz, S. (1988) Love Affairs and Differential Equations. Mathematics Magazine, 61, 35. https://doi.org/10.2307/2690328

[2] Strogatz, S. (1994) Nonlinear Dynamics and Chaos. Addison-Wesley, Boston.

[3] Sprott, J. (2004) Dynamical Models of Love. Nonlinear Dynamics, Psychology and Life Science, 8, 303-314.

[4] Deng, W., Liao, X., Dong, T. and Zhou, B. (2017) Hopf Bifurcation in a Love-Triangle Model with Time Delays. Neurocomputing, 260, 13-24.

https://doi.org/10.1016/j.neucom.2017.02.062

[5] Naoyuki, S., Sinka, I. and Taro, M. (2014) Analysis of Mathematical Model for Divorce. Research Institute for Mathematical Sciences (RIMS) Koukyuroku, Kyoto University, Kyoto, 8-17.

[6] Rinaldi, S. (1998) Laura and Petrach: An Intriguing Case of Cyclical Love Dynamics. SIAM, Journal on Applied Mathematics, 58, 1205-1221. https://doi.org/10.1137/S003613999630592X

[7] Rinaldi, S. (1998) Love Dynamics: The Case of Linear Couples. Applied Mathematics and Computation, 95, 181-192. https://doi.org/10.1016/S0096-3003(97)10081-9

[8] Rinaldi, S. and Gragnani, A. (1998) Love Dynamics between Secure Individuals: A Modeling Approach. Nonlinear Dynamics, Psychology and Life Science, 2, 283-301. https://doi.org/10.1023/A:1022935005126

[9] Rinaldi, S., Rossa, F.D. and Dercole, F. (2010) Love and Appeal in Standard Couples. International Journal of Bifurcation and Chaos, 20, 2443-2451. https://doi.org/10.1142/S021812741002709X

[10] Rinaldi, S., Rossa, F.D. and Landi, P. (2013) A Mathematical Model of "Gone with the Wind”. Physica A, 392, 3231-3239. https://doi.org/10.1016/j.physa.2013.03.034

[11] Rinaldi, S., Rossa, F.D., Dercole, F., Gragnani, A. and Landi, P. (2016) Modeling Love Dynamics. World Scientific, Singapore, World Scientific Series on Nonlinear Science Series A, 89. https://doi.org/10.1142/9656

[12] Liao, X. and Ran, J. (2007) Hopf Bifurcation in Love Dynamical Models with Nonlinear Couples and Time Delays. Chaos Solutions and Fractals, 31, 853-865. https://doi.org/10.1016/j.chaos.2005.10.037

[13] Son, W.-S. and Park, Y.-J. (2011) Time Delay Effect on the Love Dynamic Model.

[14] Akio, M. and Szidarovszky, F. (2016) Love Affairs Dynamics with One Delay in Losing Memory or Gaining Affection. Institute of Economic Research, Chuo University, Tokyo, 260, 1-23.

[15] Murray, J.D. (2002) Mathematical Biology. Third Edition, Springer, Berlin.

[16] Gopalsamy, K. (1992) Stability and Oscillations in Delay Differential Equations of Population Dynamics. Kluwer Academic Publishers, Berlin. 
https://doi.org/10.1007/978-94-015-7920-9

[17] Das, P., Roy, A.B. and Das, A. (1994) Stability and Oscillations of a Negative Feedback Delay Model for the Control of Testosterone Secretion. BioSystems, 32, 61-69. https://doi.org/10.1016/0303-2647(94)90019-1

[18] Das, P. and Roy, A.B. (1997) The Role of Four Regulatory Hormones in Controlling Testicular Function in a Delay Model. Mathematical and Computer Modelling, 25, 101-116. https://doi.org/10.1016/S0895-7177(97)00033-2

[19] Hamaya, Y., Takagi, S. and Saito, K. (2020) On the Love Dynamical Model with Delay, to Appear.

[20] Bielczyk, N., Bondnar, M. and Forys, U. (2012) Delay Can Stabilize: Love Affairs Dynamics. Applied Mathematics and Computation, 219, 3923-3937.

https://doi.org/10.1016/j.amc.2012.10.028 